\title{
Study on a New Method for Calculating Water Saturation
}

\author{
Yang Kebing, Xie Li, Zhang Lei, Li Jun, Cao Chengcheng \\ Petro China Huabei Oilfield Company, Renqiu, China \\ Email address: \\ ykbyzm123@sina.com (Yang Kebing) \\ To cite this article: \\ Yang Kebing, Xie Li, Zhang Lei, Li Jun, Cao Chengcheng. Study on a New Method for Calculating Water Saturation. International Journal of \\ Oil, Gas and Coal Engineering. Vol. 7, No. 6, 2019, pp. 125-129. doi: 10.11648/j.ogce.20190706.13
}

Received: October 20, 2019; Accepted: November 15, 2019; Published: November 25, 2019

\begin{abstract}
In order to improve the identification and evaluation accuracy of low resistivity oil layer and complex pore structure oil layer, and to avoid the interpretation error caused by the traditional evaluation mode of "high resistivity is oil layer and low resistivity is water layer", the method of calculating water saturation by radial resistivity ratio was studied. The results show that the radial resistivity ratio can be used to calculate the reservoir water saturation for completely invaded formation, which can be deduced according to archie formula. The new formula does not evaluate oil and water layers based on high or low of resistivity, but can be used to evaluate low resistivity oil and high resistivity water layers. After testing core experimental data, The new formula can effectively calculate the water saturation and meet the requirement of using. After practical application of data, The average absolute error of the calculated water saturation is $3.55 \%$, which conforms to the industry standard. This method is helpful to improve the coincidence rate of logging interpretation, and is worth popularizing.
\end{abstract}

Keywords: Radial Resistivity Ratio, Reservoir Oil Bearing, Archie's Formula, Rock-electric Experiment, Sw Calculation, Oil and Water Layer Evaluation

\section{Introduction}

Reservoir oil saturation is the key parameter of reservoir quantitative evaluation and reserves calculation, The formula was developed by Archie [1]. However, the effect is not good in argillaceous, low resistivity and complex pore reservoirs. To solve these problems, researchers have modified Archie formula by derived various extended formulas to improve the calculation accuracy of water saturation according to geological conditions [2-6]. Currently, there are nuclear magnetic logs that provide accurate water saturation data. However, due to the high cost, the calculation of water saturation mainly comes from conventional logging data [7-8] Therefore, how to use conventional logging data to improve the accuracy of water saturation calculation is still the direction of researchers' efforts [9-11].

Resistivity curve is the most critical data for calculating water saturation. Different from log curves of natural gamma ray and neutron, resistivity log has curves with different radial investigation depths. For example, induction resistivity logging has three curves: deep, medium and shallow. The array induction logging curve has five or six curves. There are also logging instruments with different design principles, such as dual lateral logging and induction logging, which provide a basis for evaluating oil and water layers with radial resistivity ratio [12-15].

For a long time, researchers have used radial resistivity to evaluate oil and water layers qualitatively, but have not used it to calculate reservoir water saturation. Further research shows that the radial resistivity ratio can be used to calculate reservoir water saturation, which requires fewer parameters than archie's formula, and the key parameters can be determined by adjacent water layers. The experimental data and practical application show that the calculated water saturation accuracy is high and can meet the application requirements.

\section{Formula Derivation and Experimental Verification of Water Saturation Calculation}

In this study, based on archie's formula and the formation invasion model, a formula for calculating the reservoir water saturation by radial resistivity ratio under the most extensive conditions was derived and verified experimentally. The detailed process is as follows: 


\subsection{Formula Derivation}

According to Archie formula, the deep resistivity and the flushing zone resistivity of reservoir in the state of full invasion can be expressed as:

$$
\begin{aligned}
\mathrm{Rt} & =\frac{a b R w}{\Phi^{m} \cdot S w^{n}} \\
\mathrm{Rxo} & =\frac{a b R m f}{\Phi^{m} \cdot S x o^{n}}
\end{aligned}
$$

The radial resistivity ratio is:

$$
\frac{\mathrm{Rt}}{R x o}=\frac{R w \cdot S x o^{n}}{R m f \cdot S w^{n}}=\frac{R w}{R m f} \cdot\left[\frac{S x o}{S w}\right]^{n}
$$

For full invasion flushing zone formations, $\mathrm{Sxo}=1$, Then the radial resistivity ratio is:

$$
\frac{\mathrm{Rt}}{R x o}=\frac{R w \cdot S x o^{n}}{R m f \cdot S w^{n}}=\frac{R w}{R m f} \cdot \frac{1}{S w^{n}}
$$

The water saturation is:

$$
\mathrm{Sw}=\sqrt[n]{\frac{R w / R m f}{R t / R x o}}
$$

The above equation is a new formula for calculating reservoir water saturation using radial resistivity ratio.

Where (In equations 1 to 5): Rt is deep resistivity of reservoir, Rxo is flush zone resistivity after complete invasion, $\mathrm{Rw}$ is formation water resistivity, $\mathrm{Rmf}$ is mud filtrate resistivity, $\Phi$ is reservoir porosity, a, b, m, n is rock-electric experiment parameter, $\mathrm{Sw}$ is water saturation, Sxo is flush zone water saturation.

An important prerequisite for the above deduction is that the water saturation of the flushing zone is 1 , that is, the flushing zone is completely invaded. The flushing zone refers to a circular belt around the wellbore formed after the movable fluid in the formation is completely displaced by the mud filtrate due to the intrusion of mud in the wellbore, with a radial depth of about $10 \mathrm{~cm}$. According to the formation invasion model, the mud filtrate invasion into oil, gas and water can be divided into three stages, as shown in Figure 1.

In the first section near the shaft wall, that is, the flushing zone, the mud filtrate has the highest invasion degree, which is basically considered to be complete invasion. In the second stage, mud filtrate intrusion interacts with the undisturbed formation fluid, and is the area where mud filtrate intrusion can affect, called intrusion zone or transition zone. In the third section, The mud filtrate penetrates the area where it cannot be affected, That is undisturbed zone. Therefore, according to the actual drilling situation, it is the overwhelming majority that the flushing zone of oil, gas and water layer is completely invaded, which indicates that the assumption that the water saturation of the flushing zone is 1 is feasible in calculation.
It can be seen that in the new formula, there are only three parameters, Rw, Rmf and n, which are all constant terms. This indicates that there is a power function relationship between water saturation and the ratio of deep resistivity to flushing zone resistivity, indicating that the oil-bearing property of reservoir is positively correlated with the ratio of deep resistivity to flushing zone resistivity. When the water saturation value is the maximum, the resistivity ratio of deep and flush zone is the minimum. When water saturation decreases, the resistivity ratio of deep and flush zone increases. That is to say, whether drilling with fresh water mud or salt water mud, the resistivity ratio in deep and flush zone has the same characteristics to reflect the oil-bearing reservoir. The greater the resistivity ratio is, the better the oil-bearing reservoir is; the smaller the resistivity ratio is, the worse the oil-bearing reservoir is. Therefore, the ratio of the deep resistivity to the flushing zone resistivity can reflect the oil-bearing of the reservoir when the flushing zone is completely invaded.

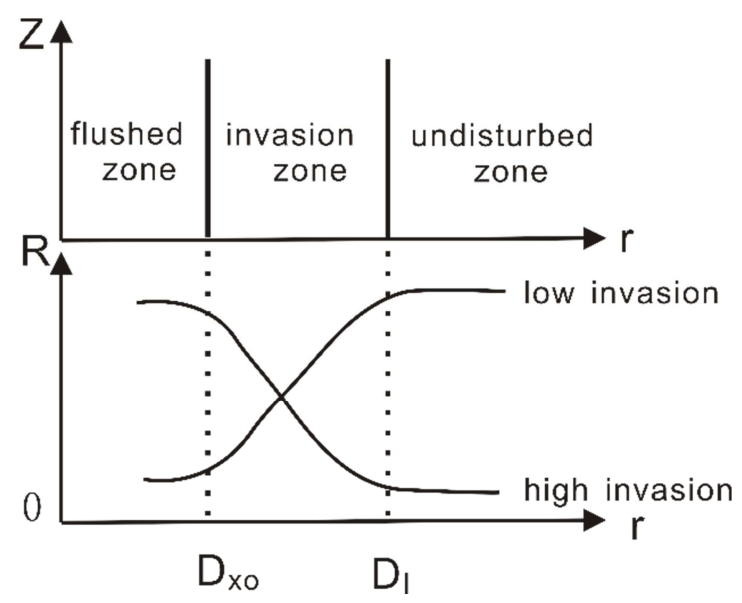

Figure 1. Formation intrusion model and radial resistivity profile.

\subsection{Verification of Experimental Data}

The reliability of the calculation formula of water saturation is tested by multi-block core experiments. Firstly, the core was soaked with $3000 \mathrm{mg} / \mathrm{L}$ salt water to simulate the resistivity of the mud filtrate of the flushing zone completely invaded, and the core resistivity was measured when the water saturation was $100 \%$, which was used as the resistivity of the reservoir flushing zone. Secondly, the core was soaked in $8000 \mathrm{mg} / \mathrm{L}$ salt water to simulate the resistivity of undisturbed formation water, and then displaced by kerosene. Core resistivity and water saturation were measured when different displacement degrees were recorded to be regarded as the true resistivity of reservoirs with different water saturations under undisturbed formation water conditions. According to the proposed new water saturation formula (5), the reservoir water saturation is calculated. The comparison between the calculated results and the measured values is shown in table 1. 
Table 1. Comparison of core experiment and reservoir water saturation calculation results.

\begin{tabular}{|c|c|c|c|c|}
\hline $\begin{array}{l}\text { measure water } \\
\text { saturation } / \%\end{array}$ & $\begin{array}{l}\text { simulated undisturbed formation } \\
\text { resistivity } / \Omega \cdot m\end{array}$ & 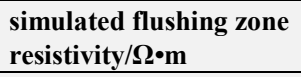 & $\begin{array}{l}\text { Calculate water } \\
\text { saturation } / \%\end{array}$ & $\begin{array}{l}\text { Absolute } \\
\text { error }\end{array}$ \\
\hline 46.0 & 163.3 & 85.5 & 47.3 & -1.3 \\
\hline 39.0 & 122.5 & 52.4 & 37.4 & 1.6 \\
\hline 32.0 & 54.1 & 19.5 & 36.5 & -4.5 \\
\hline 30.0 & 114.5 & 35.1 & 31.7 & -1.7 \\
\hline 26.0 & 57.1 & 18.2 & 23.3 & 2.7 \\
\hline
\end{tabular}

It can be seen that the maximum absolute error of water saturation calculated by equation (5) and the actual core measurement of water saturation is $4.5 \%$, the minimum absolute error is $1.3 \%$, and the average absolute error is $2.4 \%$. The accuracy meets the industry requirements of well logging calculation of water saturation. Therefore, when the formula requires parameters $\mathrm{n}$ and $\mathrm{Rw} / \mathrm{Rmf}$ to be effectively determined, the reservoir water saturation calculated by the new formula (5) can be used in production practice.

\section{Parameters Obtained}

There are three parameters in the formula, $\mathrm{Rw}, \mathrm{Rmf}$ and $\mathrm{n}$, which need to be determined. Among them, Rw and Rmf only need the ratio $\mathrm{Rw} / \mathrm{Rmf}$, and do not need their specific values. Of course, it is better to have specific values of Rw and Rmf, and the effect is the same as the acquired ratio of Rw/Rmf. As can be seen, only two parameters need to be determined in practical application, namely, $\mathrm{Rw} / \mathrm{Rmf}$ value and $\mathrm{n}$ value.

\subsection{Parameter Rw/Rmf Obtained}

Under the condition of complete invasion, the ratio of formation water resistivity to mud filtrate resistivity is exactly equal to the ratio of water layer resistivity to flush zone resistivity. This rule can be proved by Archie formula.

According to Archie formula, the resistivity logging response equation of water layer completely invaded is:

$$
\begin{aligned}
& \text { Ro }=\frac{a R w}{\Phi^{m}} \\
& \mathrm{Rxo}=\frac{a R m f}{\Phi^{m}}
\end{aligned}
$$

Then Rw/Rmf is:

$$
\frac{R w}{R m f}=\frac{\mathrm{Ro}}{R x o}
$$

Where (In equations 6 to 8): Ro is deep resistivity of water layer, Rxo is flush zone resistivity of water layer after complete invasion, $\mathrm{Rw}$ is formation water resistivity, $\mathrm{Rmf}$ is mud filtrate resistivity, $\Phi$ is reservoir porosity, a, $\mathrm{m}$ is rock-electric experiment parameter.

It can be seen that, for pure water layer completely invaded, the ratio of deep detection resistivity and flushing zone detection resistivity is theoretically a constant, that is, the ratio of undisturbed formation water resistivity and mud filtrate resistivity $\mathrm{Rw} / \mathrm{Rmf}$. Due to deposition, the resistivity of undisturbed formation water is basically stable under an oil-water system, and the resistivity of drilling mud filtrate is also basically stable for the same well. Therefore, the ratio is also stable, which is basically a constant. Through the investigation of the actual logging data, the ratio of the pure water layer deep resistivity to flushing zone resistivity changes little and is basically a constant. For the need of calculation, the smallest one is taken as the calculation parameter.

\subsection{Obtained of Parameter $n$}

The parameter $\mathrm{n}$ is the saturation index, which can be obtained from the experimental data of rock electricity. Since all the old oil field blocks have been carried out rock-electric experimental research at present, the rock-electric experimental measurement data can be directly used for parameter $n$. In exploration new areas without rock-electricity experimental data, regional empirical parameters can be used for calculation.

At the same time, predecessors have conducted a lot of studies on the influencing factors of saturation index $n$, believing that the saturation index is mainly related to reservoir formation water salinity, porosity, permeability, pore structure, etc., and put forward some methods to determine the saturation index. For example, the saturation index $\mathrm{n}$ can be obtained by using formation water resistivity and saturation index regression.

\section{Application Example of Core Data Well}

Figure 2 is an example of a well with core measurement water saturation data in a certain block (well xx6-5). The water saturation data used is mercury injection experiment irreducible water saturation data, which can represent the ideal water saturation when the reservoir is full of oil and gas. The resistivity data are array induction. $\mathrm{R} 9$ is used to represent the undisturbed formation resistivity, $\mathrm{R} 1$ is used to represent the resistivity of the completely invasive flushing zone, $\mathrm{Rw} / \mathrm{Rmf}$ value is determined according to the R9/R1 value of the typical adjacent water layer, and $n$ value is determined by the rock-electric experimental data. The new formula was adopted to processing the well, and it can be seen that the water saturation calculated in the target interval 2230-2270 is basically consistent with the trend of irreducible water saturation measured in the experiment, with the highest water saturation of $49.2 \%$ and the lowest water saturation of $29.6 \%$, meeting the reservoir standards. In this oil test, the daily oil is $17.1 \mathrm{~m}^{3}$, the daily gas is $262,800 \mathrm{~m}^{3}$, and the daily water is a small amount. It is a condensate gas layer. The processing results are consistent with the oil test results. The maximum 
absolute error is $13.8 \%$, the minimum absolute error is $0.1 \%$, and the average absolute error is $3.55 \%$. Its precision can meet

the use requirements.

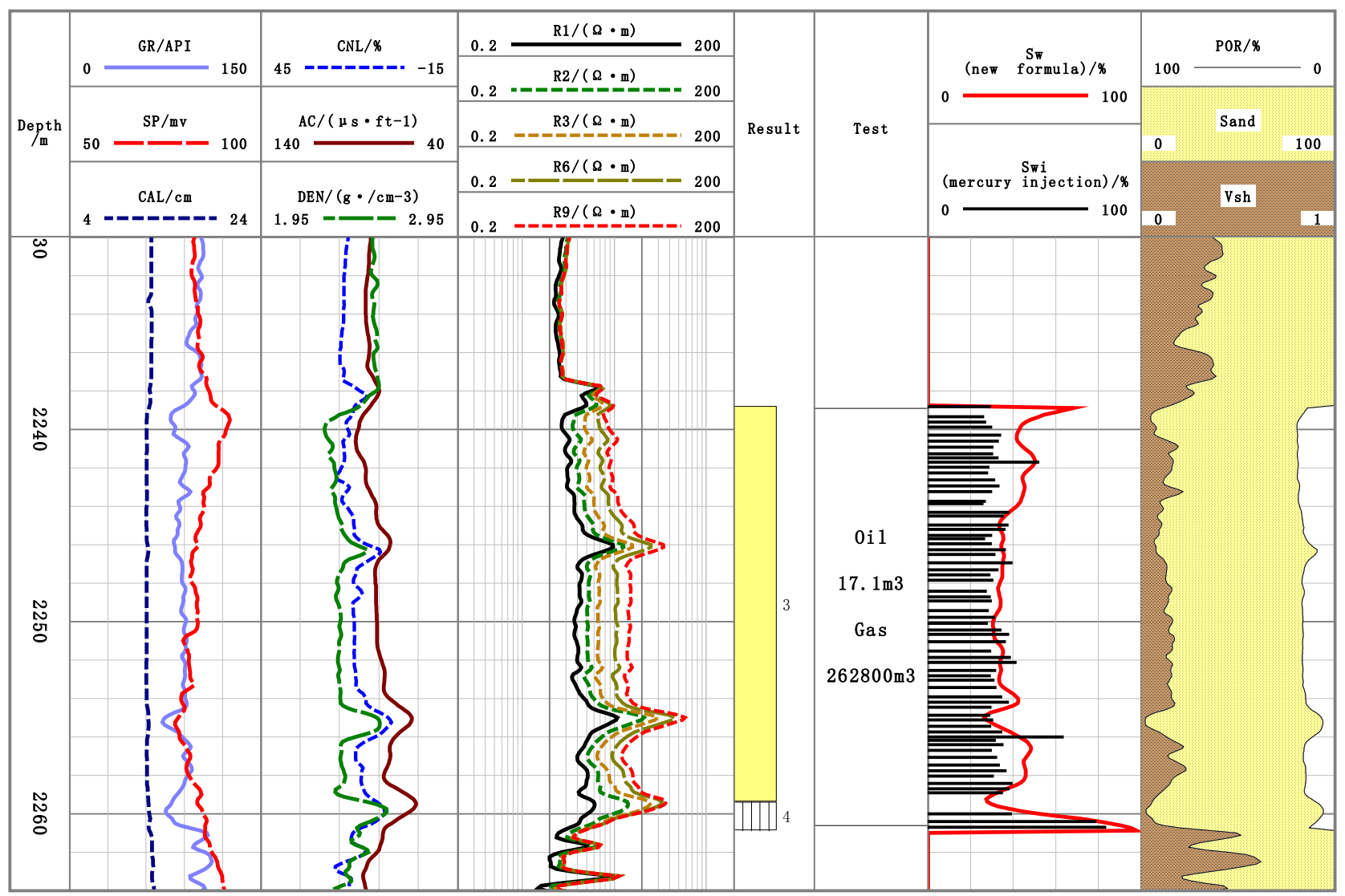

Figure 2. Sw treatment result of new formula of well $x x 6-5$ in a block.

In addition, the new formula is used to calculate the water saturation. Since the deep and shallow resistivity ratio of pure water layer is used to calibrate the calculation results, the calculation of water saturation is more reasonable. No case is greater than $100 \%$, which avoids the previous case of $\mathrm{Sw}>100 \%$ caused by improper parameter selection. At the same time, complex pore reservoirs with poor physical properties, such as poor oil layer and dry layer, are treated with the same parameters as oil layer, which avoids unreasonable calculation problems caused by previous parameter value errors and improves the calculation accuracy of water saturation.

\section{Conclusions}

The relationship between the radial resistivity ratio and reservoir water saturation is a power function. The greater the radial resistivity ratio is, the better the oil bearing of the reservoir is; the smaller the radial resistivity ratio is, the worse the oil bearing of the reservoir is, There is a one-to-one correspondence between them. It can be used for oil layer and water layer evaluation.

Using radial resistivity ratio to calculate reservoir water saturation can eliminate the influence of reservoir lithology and physical property changes on the calculation of water saturation. This method can effectively evaluate low resistivity oil and high water layers. It provides a new method for the evaluation of difficult logging layers.

Through core test data verification and single well digital processing verification, the calculation results of the new formula can meet the requirements of the logging industry, and avoid unreasonable calculation problems caused by the parameter error of archie formula, which is helpful to improve the accuracy rate of logging interpretation, and is worthy of further popularization.

\section{References}

[1] Archie, G. E. The electrical resistivity $\log$ as an aid in determining some reservoir characteristics [J]. Tnas Am Inst MechEng, 1942, 146: 54-61.

[2] Pickett, G. R. A review of current techniques for determination of water saturation from Logs $[\mathrm{J}]$. Journal of Petroleum Technology, 1966, 18 (11): 1425-1433.

[3] Clavier, C., Coates, G., Dumanoir, J. Theoretical and experimental bases for the dual-water model for interpretation of shaly sands [J]. Society of Petroleum Engineers Journal, 1984. 24 (2): 153-168.

[4] Zhang, C. G., Mao, Z. Q., Ou, Y. J. The New technique for evaluating water saturation of the "low resistivity" reservoir In J Field [C]. SPWLA 35th Annual Logging Symposium, 1994, 19-22, June, Tulsa, Oklahoma. 
[5] Hamada, G. M., Alawad, M. Petrophysical Evaluation of Low Resistivity Sandstone Reservoirs [J]. Journal of Canadian Petroleum Technology, 2000, 39 (7).

[6] Koelman, J. M., Fanini, O. N., Kreigshauser, B. F. Enhanced evaluation of low-resistivity reservoirs using multi-component induction log data $[\mathrm{J}]$. Society of Petrophysicists and Well-Log Analysts, 2001, 42 (6).

[7] Wen Tang. One Well Evaluation in Low Permeability Gas Sandstones from Nuclear Magnetic Resonance (NMR) logs [C]. SPE Middle East Unconventional Gas Conference and Exhibition, 2011, 31 January-2 February, Muscat, Oman.

[8] Zakir Hossain. A relationship between rock physics and nuclear magnetic resonance [C]. SEG International Exposition and Annual Meeting, 2016, 16-21 October, Dallas, Texas.

[9] Verga, F., Giaccardo, A., Gonfalini, M. Determination of uncertainties in water saturation calculations from well log data using a probabilistic approach [C]. Offshore Mediterranean Conference and Exhibition, 2001, 28-30 March, Ravenna, Italy.

[10] Leandro, H. M., Alexandre, C. V., Ivan, M. F., et al. The impact of Archie's parameters in the calculation of water saturation for carbonate reservoir, Campos Basin, Brazil [C]. SEG Annual Meeting, 2015, 18-23 October, New Orleans, Louisiana.
[11] Sarihi, A., Vargas-Murillo, B. A method to compute water saturation in tight rocks accounting for conductivity of clay minerals [C]. Abu Dhabi International Petroleum Exhibition and Conference, 2015, 9-12 November, Abu Dhabi, UAE.

[12] Yu Hongyan, Wei Xiaolong, Wang Zhenliang, et al. Review of water saturation calculation methods in shale gas reservoir [C]. SPE Asia Pacific Oil and Gas Conference and Exhibition, 2018, 23-25 October, Brisbane, Australia.

[13] Deng Shaogui, Sun Qingtao, Li Hu, et al. The sensitivity of the array resistivity $\log$ to mud filtrate invasion and its primary five-parameter inversion for improved oil water recognition [J]. Petroleum Science, 2012, 9 (6): 295-302.

[14] Yang Kebing, Yuan Xiaohong, Liu Mingbo, et al. Resistivity ratio method to evaluate oil-water layer [J]. Natural Gas Exploration \& Development, 2015, 38 (1): 37-39.

[15] Yang Kebing, Li Shidong, Wang Huijing, et al. Discussion on the method of evaluating oil and water layers by using ratio of deep resistivity and flush zone resistivity [J]. International Journal of Oil, Gas and Coal Engineering, 2019, 7 (1): 7-12. 